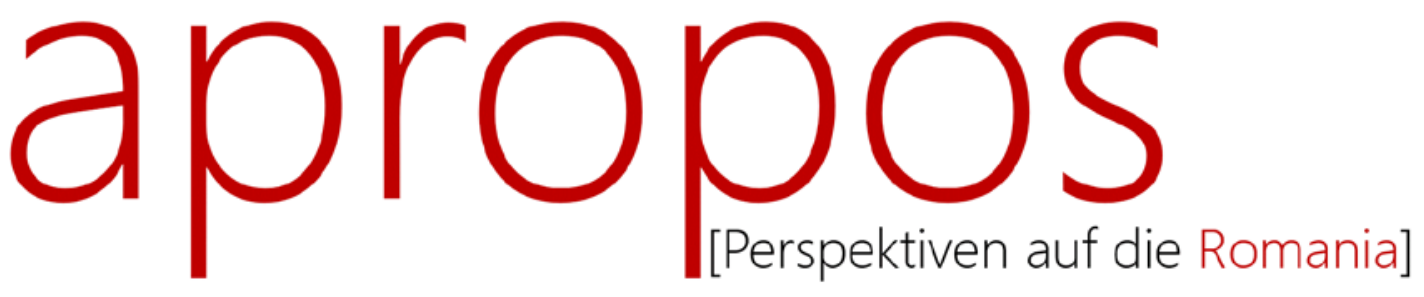

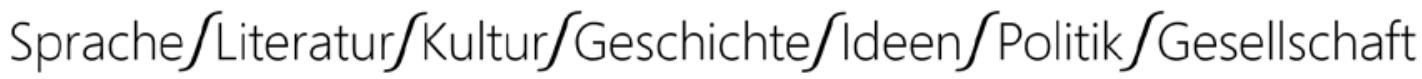

$5 \mid 2020$

Außenseiterdiskurse - interdisziplinäre Perspektiven auf ein anhaltend aktuelles Phänomen

Rezension von MARCHI, Riccardo. 2018. The Portuguese Far Right. Between Late Authoritarianism and Democracy (1945-2015). London: Routledge.

Teresa Pinheiro

apropos [Perspektiven auf die Romania]

hosted by Hamburg University Press

STAATS- UND UNIVERSITÄTS-

BIBLIOTHEK

HAMBURG

2020, 5

pp. $173-176$

CARL VON OSSIETZKY

ISSN: $2627-3446$

Online

https://journals.sub.uni-hamburg.de/apropos/article/view/1589

Zitierweise

Pinheiro, Teresa. 2020. „Rezension von MARCHI, Riccardo. 2018. The Portuguese Far Right. Between Late Authoritarianism and Democracy (1945-2015). London: Routledge", apropos [Perspektiven auf die Romania] 5, 173-176. doi:

10.15460/apropos.5.1589

Except where otherwise noted, this article is licensed under a Creative Commons Attribution 4.0 International license (CC BY 4.0)

\title{
cccreative commons (i)
}




\title{
Theresa Pinheiro
}

\section{Rezension}

\section{MARCHI, Riccardo. 2018. The Portuguese Far Right. Between Late Authoritarianism and Democracy (1945-2015). London: Routledge.}

\author{
Teresa Pinheiro \\ ist Inhaberin der Professur Kultureller \\ und Sozialer Wandel am Institut für \\ Europäische Studien und \\ Geschichtswissenschaft der \\ Technischen Universität Chemnitz \\ teresa.pinheiro@phil.tu-chemnitz.de
}

\section{Keywords}

Rechtsextremismus - Portugal - 20. Jahrhundert - 21. Jahrhundert

Lange Zeit galt das westlichste Land Kontinentaleuropas als die letzte Insel der Glückseligen: Als einziger Staat in Europa sei Portugal vom gegenwärtigen Einzug rechtsextremer Parteien in die Politik verschont geblieben. Doch war dies nur eine Frage der Zeit, wie die letzten Parlamentswahlen im Oktober 2019 gezeigt haben. Seitdem ist die rechtspopulistische Partei Chega! („Es reicht!“) mit einem Abgeordneten im portugiesischen Parlament vertreten. In den azorischen Regionalwahlen vom 25. Oktober 2020 erzielte die Partei sogar zwei Sitze und könnte eine Schlüsselrolle bei der Ermöglichung einer konservativen Regierung spielen.

Für Portugal-Kenner*innen kommt diese Entwicklung nicht überraschend. Nicht nur blickt der Rechtsextremismus in Portugal auf eine lange Tradition zurück, sondern waren auch in der demokratischen Ära nach 1974 rechtsextremistische Gruppierungen aktiv. Zwar sind diese randständig, durch Anwendung von Gewalt gegen Immigranten ziehen sie jedoch gelegentlich die mediale Aufmerksamkeit auf sich. Das Novum an Chega! ist der Einzug einer rechtsextremen Partei in die höchste Kammer demokratischer Volksvertretung. Unklar ist gegenwärtig noch, ob die Partei die Wirkungskraft besitzt, als Katalysator für bestehende Probleme zu fungieren. Konnte die spanische Vox aus dem territorialen Konflikt um Katalonien Kapital schlagen, konnte die AfD in Deutschland die Flüchtlingskrise für die eigenen Zwecke instrumentalisieren, könnte Chega! zum politischen Katalysator für den in Portugal allgegenwärtigen kolonialbedingten Rassismus werden - noch am vergangen 25. Juli ist der Schauspieler Bruno Candé in einem Vorort von Lissabon auf offener Straße von einem Veteranen des Kolonialkriegs ermordet worden. 
Angesichts dieser Aktualität könnte die Veröffentlichung von Riccardo Marchis The Portuguese Far Right. Between Late Authoritarianism and Democracy (1945-2015) im Jahr 2019 kaum passender sein. Zwar befasst sich das Buch nicht mit der Partei Chega!, die erst 2019 gegründet wurde, dennoch lädt es ein, diese und andere gegenwärtige Tendenzen aus dem rechtsextremen Spektrum im Lichte der Zeitgeschichte Portugals zu betrachten. Die Studie setzt in der Nachkriegszeit an, was nicht bedeutet, dass es zuvor keine rechtsextremen Tendenzen in Portugal gegeben hätte - im Gegenteil: Gerade in der ersten Hälfte des 20. Jahrhunderts hat es unterschiedliche Reaktionen auf das Ende der Monarchie und die Etablierung der Ersten Republik (1910-1926) gegeben, die von den streng nationalistischen Positionierungen des Integralismus und Regenerationismus bis hin zu nationalsyndikalistischen Bewegungen reichten. Dass Marchi seine Chronologie nicht zu Beginn des 20. Jahrhunderts ansetzt, sondern erst nach 1945 liegt also nicht an der Abwesenheit rechtsextremer Tendenzen vor dem Zweiten Weltkrieg. Vielmehr ergibt sich die Schwerpunktsetzung aus der Berücksichtigung der Forschungslandschaft: Von den wenigen Studien zum Rechtextremismus in Portugal beziehen sich die meisten auf die Zeit der Ersten Republik und der Anfänge des Estado Novo. Mit der Gewichtung der diachronen Analyse auf die 70 Jahre seit dem Ende des Zweiten Weltkriegs bis in die Gegenwart deckt das Buch wichtige Epochen der portugiesischen Zeitgeschichte ab - von der kosmetischen Bereinigung faschistischer Züge des Estado Novo in der direkten Nachkriegszeit und der politischen Öffnung unter Marcelo Caetano über die revolutionären Zeiten nach der Nelkenrevolution bis hin zur späteren politischen Normalisierung und Annäherung an Europa. In der Schließung dieses Forschungsdesiderats liegt der Hauptvorzug dieser Arbeit.

Getreu der Zielsetzung, die Entwicklung der rechtsextremen Tendenzen im Kontext der Zeitgeschichte Portugals nachzuzeichnen, ist das Buch chronologisch in drei Teile aufgeteilt: Der erste Teil behandelt die Zeit vom Ende des Krieges bis zur Nelkenrevolution (1945-1974), der zweite Teil geht auf die Phase der Transition zur Demokratie ein (1974-1982) und der dritte Teil ist der Epoche der konsolidierten Demokratie gewidmet (1982-2015). Mit dieser Einteilung bettet Marchi seinen Gegenstand nachvollziehbar in eine klassische Periodisierung der Geschichte Portugals ein. Weniger nachvollziehbar ist, warum fast dreißig Jahre Diktatur als ihr Ende verstanden werden - „Part I: The far right at the end of the authoritarian regime (1945-1974)". Dies wird nicht weiter erläutert und überzeugt nicht. Sieht man die Herausforderungen des Estado Novo in der Nachkriegszeit - Akzeptanz im Westen, Kolonialkrieg, internen politischen Widerstand - als einen vordeterminierten Weg in den Sturz, so müsse die portugiesische Diktatur als ein von Beginn an totgeweihtes, in ewiger Agonie befindliches Regime angesehen werden.

Der erste Teil des Buches ist in drei Kapitel unterteilt, die mit drei unterschiedlichen Phasen der Diktatur korrespondieren. Im ersten Kapitel geht der Autor auf die Zeit zwischen 1945 und 1960 ein. Portugals Neutralität im Konflikt und seine Annäherung an die Alliierten ab 1943 war ein strategischer Schachzug Salazars, der Portugals Anschluss an den Westblock in der Nachkriegszeit ermöglichte. Teil dieser Strategie war die Entpolitisierung der Massenorganisationen. Dies brachte einen 
anfänglichen Aufruhr von seit den 1930er Jahren aktiven germanophilen und rechtsradikalen Gruppen mit sich, die aber gegen Ende der fünfziger Jahre fast vollständig neutralisiert waren. Das zweite Kapitel befasst sich mit der Zeit von 1961 bis 1968, in der der Beginn des Kolonialkriegs 1961 und die studentischen Proteste von 1962 eine kurzzeitige Erneuerung politischer Aktivisten aus dem rechtsextremen Spektrum begünstigten, allen voran Studierende, die in diesen Ereignissen einen Beweis der Schwäche des Regimes sahen. Die Amtszeit von Salazars Nachfolger Marcelo Caetano (1968-1974) gibt die chronologische Klammer des dritten Kapitels. Die politischen Öffnungstendenzen der Caetano-Ära waren ein Ventil für die Protestbewegungen der sechziger Jahre, die nun eindeutig und offen die Demokratisierung und Entkolonialisierung des Landes forderten. Zugleich riefen sie jedoch die Reaktion der Ultrarechten auf die politische Bühne.

Im zweiten Teil geht der Autor auf die rechtsextremen Bewegungen im Kontext der demokratischen Transition ein. Das erste Kapitel ist der bewegten und von der Hegemonie linker Parteien dominierten revolutionären Periode von 1974 und 1975 gewidmet. Angesichts der Dämonisierung der Rechten während dieser revolutionären Zeiten, sahen sich viele rechte Aktivisten gezwungen, ins Ausland zu gehen, um Lustrationsprozessen oder gar Freiheitsstrafen zu entkommen. Viele von ihnen fanden im noch-franquistischen Madrid nicht nur einen sicheren Hafen, sondern auch ein ergiebiges Netz der ultrarechten Franquisten zu denen Blás Piñar mit Fuerza Nueva gehörte. Dennoch konnten sich einige antikommunistische und antimarxistische Gruppen im Untergrund etablieren, die zwischen Mai 1975 und März 1976 über 400 terroristische Anschläge verbuchten, die fast immer Sitze und Organe der Kommunistischen Partei zum Ziel hatten. Die politische Normalisierung, die mit der Vereitelung des revolutionären Putschversuchs vom 25. November 1975 und der darauffolgenden Schwächung der Kommunistischen Partei begann, begünstigte die Auflösung der terroristischen Gruppen aus dem rechten Spektrum zugunsten der politischen Institutionalisierung. Diesen meist gescheiterten Versuchen der Etablierung einer Partei rechts von der christlich-konservativen CDS zwischen 1976 und 1982 ist das zweite Kapitel gewidmet. Das dritte und letzte Kapitel des zweiten Teils geht auf die Zeit von der Verfassungsreform von 1982, die das Ende der revolutionären Zeit in Portugal endgültig besiegelte, bis zur Unterzeichnung des EWG-Beitrittsvertrags im Juni 1985 ein. In dieser Zeit formierte sich in Portugal eine neue Generation des Rechtsextremismus, die keine Kontinuität zum Estado Novo aufwies, sondern an antiliberale New-Right-Tendenzen aus den USA und Großbritannien anknüpfte.

Der dritte und letzte Teil des Buches behandelt die Zeit der konsolidierten Demokratie seit 1982. Das erste Kapitel analysiert die seit den 1980er Jahren aktiven Gruppen von Neonazis und Skinheads im Kontext der Immigration aus den ehemaligen Kolonien und den Ausbruch rassistischer und fremdfeindlicher Angriffe gegen Einwanderer insbesondere in den Peripherien Portos und Lissabons. Das zweite Kapitel greift neue Tendenzen der politischen Organisation des Rechtsextremismus auf. Besondere Aufmerksamkeit wird der Etablierung der Nationalen Erneuerungspartei (PNR) geschenkt, die noch heute unter der Bezeichnung Erguete („Steh auf“) existiert, obwohl sie nie parlamentarische Repräsentation erlangte. 
Das letzte Kapitel geht auf jüngste Tendenzen des Rechtsextremismus im gegenwärtigen Portugal ein. Im Zentrum des Kapitels stehen identitäre Bewegungen, die im Jahre 2001 mit der Organisation des ersten Nationalistischen Kongresses in Lissabon zum ersten $\mathrm{Mal}$ in der Öffentlichkeit sichtbar wurden. Marchi bettet diese jüngsten Tendenzen des Ethnonationalismus in Portugal in den europäischen Kontext ein. Vor allem französische identitäre Bewegungen wie Terre et Peuple und Ideologen wie Alain de Benoist haben einen nachhaltigen Einfluss auf die portugiesischen Identitären ausgeübt, die mittlerweile mit der Gruppe Causa Identitária in den Social Media präsent sind.

Riccardo Marchis The Portuguese Far Right ist ein wichtiges Buch für all diejenigen, die sich für die zwar residualen, aber existierenden Tendenzen des Rechtsextremismus in Portugal interessieren und nicht des Portugiesischen mächtig sind. Diese Tendenzen werden im Kontext der jeweiligen Epochen der portugiesischen Geschichte seit dem Zweiten Weltkrieg betrachten, was ihren Charakter und ihre Motive nachvollziehbar macht. Dieser ist einer der Vorzüge dieser Arbeit, gepaart mit dem informativen Gehalt. Der Autor ist um Vollständigkeit bemüht und liefert viele Informationen über Namen, Publikationen und Aktivitäten auch von Untergrundorganisationen. Diese Vorzüge machen zugleich die Schwäche des Buches aus. Zum einen verstrickt sich der Autor in seiner eigenen Bemühung um Chronologie. Vor allem im zweiten Teil des Buches werden Phasen der rechtsextremen Bewegungen unterschieden, die sich nicht nur der allgemeinen Chronologie des Buches widersetzen, sondern auch im Laufe des Kapitels nicht weiterverfolgt werden. Zum anderen - und mit dieser Obsession für die Chronologie zusammenhängend - bleibt diese Studie hauptsächliche deskriptiv. Eine theoretische Einbettung in die sehr ergiebige politikwissenschaftliche Extremismusforschung fehlt hier fast vollständig. Auch die theoriegetränkte Verwendung der Begrifflichkeit wird hier kaum problematisiert, sieht man von einem Vierzeiler $a b$, in dem für die undifferenzierte Verwendung der Begriffe extreme right, right-wing-radicalism und radical right plädiert wird. Die mangelnde theoretische Reflexion mündet in der analytischen Schwäche des Buches, das mangels einer sauberen Begrifflichkeit ein konfuses Bild ergibt, das für ein mit der portugiesischen Parteilandschaft wenig vertrautes Publikum zur fehlgeleiteten Interpretationen führen kann. Ein im romanischen Sprachstil haftender Duktus und die unzureichende Revision des Manuskripts erschweren das Lesen und führen zu missverständlichen Aussagen (etwa die Behauptung auf S. 101, dass die zweite Phase der demokratischen Transition von rechtsextremen Gruppen vorangetrieben wurde). Schließlich ist auch ein recht freier Umgang mit Nachweisen zu monieren. Viele und wichtige bis umstrittene Behauptungen werden nicht im Dialog mit der Sekundärliteratur diskutiert, es fehlen bibliographische Nachweise und es wird insgesamt wenig auf die vorhandene Forschung verwiesen. Dies ist umso wichtiger, da es sich um eine Studie handelt, die auf Sekundärliteratur und nicht auf Archivalien basiert. 